\title{
Risk of low bone mineral density and low body mass index in patients with non-celiac wheat- sensitivity: a prospective observation study
}

Antonio Carroccio ${ }^{1,2^{*}}$, Maurizio Soresi ${ }^{1 \dagger}$, Alberto D'Alcamo ${ }^{1 \dagger}$, Carmelo Sciumè ${ }^{3 \dagger}$, Giuseppe lacono ${ }^{4 \dagger}$, ${\text { Girolamo Geraci }{ }^{3 \dagger} \text {, Ignazio Brusca }}^{5 \dagger}$, Aurelio Seidita ${ }^{1 \dagger}$, Floriana Adragna ${ }^{1 \dagger}$, Miriam Carta ${ }^{1 \dagger}$ and Pasquale Mansueto ${ }^{1+}$

\begin{abstract}
Background: Non-celiac gluten sensitivity (NCGS) or 'wheat sensitivity' (NCWS) is included in the spectrum of gluten-related disorders. No data are available on the prevalence of low bone mass density (BMD) in NCWS. Our study aims to evaluate the prevalence of low BMD in NCWS patients and search for correlations with other clinical characteristics.

Methods: This prospective observation study included 75 NCWS patients (63 women; median age 36 years) with irritable bowel syndrome (IBS)-like symptoms, 65 IBS and 50 celiac controls. Patients were recruited at two Internal Medicine Departments. Elimination diet and double-blind placebo controlled (DBPC) wheat challenge proved the NCWS diagnosis. All subjects underwent BMD assessment by Dual Energy X-Ray Absorptiometry (DXA), duodenal histology, HLA DQ typing, body mass index (BMI) evaluation and assessment for daily calcium intake.
\end{abstract}

Results: DBPC cow's milk proteins challenge showed that 30 of the 75 NCWS patients suffered from multiple food sensitivity. Osteopenia and osteoporosis frequency increased from IBS to NCWS and to celiac disease (CD) ( $P$ <0.0001). Thirty-five NCWS patients (46.6\%) showed osteopenia or osteoporosis. Low BMD was related to low BMI and multiple food sensitivity. Values of daily dietary calcium intake in NCWS patients were significantly lower than in IBS controls.

Conclusions: An elevated frequency of bone mass loss in NCWS patients was found; this was related to low BMI and was more frequent in patients with NCWS associated with other food sensitivity. A low daily intake of dietary calcium was observed in patients with NCWS.

Keywords: Non-celiac wheat sensitivity, Multiple food allergy, Body mass index, Osteoporosis

\section{Background}

Celiac disease $(C D)$ has been reported to increase the risk of osteoporosis, with a resulting augmented risk of fractures [1]. More recently, it has been reported that a consistent percentage of the general population consider themselves to be suffering from problems caused by wheat and/or gluten ingestion, even though they do not have $\mathrm{CD}$ or wheat allergy $[2,3]$. This clinical condition

\footnotetext{
* Correspondence: acarroccio@hotmail.com

${ }^{\dagger}$ Equal contributors

'Department of Internal Medicine, University Hospital of Palermo, Via del Vespro 141 , Palermo, Italy

2Department of Internal Medicine, Giovanni Paolo II Hospital, Via Pompei, Sciacca, Italy

Full list of author information is available at the end of the article
}

has been named Non-Celiac Gluten Sensitivity' (NCGS). In a previous paper [4] we suggested the term 'Non-Celiac Wheat Sensitivity' (NCWS), since it is not known what component of wheat causes the symptoms in NCGS patients, and we also showed that these patients had a high frequency of coexistent multiple food hypersensitivity [4]. That previous study also showed a percentage of NCWS patients had weight loss and anemia: whether these depended on the intestinal malabsorption or not remains unclear.

As yet no data are available on the presence and prevalence of low bone mass density (BMD) in NCWS patients. The aims of the present study were: 1 ) to investigate the prevalence of low BMD in NCWS patients; 
and 2) to search for a possible correlation between BMD and other clinical characteristics, in particular the body mass index (BMI), of NCWS patients.

\section{Methods}

\section{Study population}

This prospective study included consecutive adult patients with an irritable bowel syndrome (IBS)-like clinical presentation, according to Rome II criteria [5], and a definitive diagnosis of NCWS. The patients were recruited at the Internal Medicine Department of the University Hospital of Palermo and at the Internal Medicine Department of the Hospital of Sciacca (Italy), between July 2011 and July 2013. Most of the patients were referred due to intestinal symptoms with onset after wheat ingestion. None of them were included in previous published studies.

NCWS diagnosis was made according to the recently proposed criteria [2] (see Additional file 1). Both the CD specific serum antibodies and the duodenal histology were evaluated on a diet containing at least 100 grams of wheat per day, for at least three months. Furthermore, according to our experience [4], all patients met the following adjunctive inclusion criteria: 1) resolution of the IBS symptoms on a standard elimination diet excluding wheat, cow's milk, egg, tomato, chocolate and other self-reported food(s) causing symptoms; and 2) symptom reappearance on double-blind placebo-controlled (DBPC) wheat challenge. DBPC cow's milk protein (CMP) challenge and other 'open' food challenges were also performed in all cases [4].

Exclusion criteria were: 1) self-exclusion of wheat from the diet and refusal to reintroduce it, before entering the study; 2) nervous system disease, major psychiatric disorder; 3) hyperparathyroidism, Cushing's syndrome, kidney disease and other 'organic' gastrointestinal disorders; 4) physical impairment limiting physical activity; 5) menopause; and 6) steroid and sex steroid therapy, hormone replacement therapy or ovariectomy.

Fifty sex- and age-matched subjects with CD and sixtyfive suffering from IBS unrelated to NCWS or other food 'intolerance,' diagnosed according to standard criteria during the same study period, were chosen at random (randomized by computer generated method among the patients with a new CD or IBS diagnosis posed during the same study period) and enrolled as control groups. The same exclusion criteria used for the NCWS patients were applied to the control groups.

\section{Methods}

On entering the study all patients underwent serum assays and HLA-DQ typing for CD diagnosis (see Additional file 1). Specific IgE (RAST) and/or skin prick tests for food allergens were performed on all patients, as previously described [4].

\section{Duodenal histology}

Four to six biopsy specimens were obtained from the bulb and the second duodenal portion during gastroduodenoscopy, performed when the patients were on a wheat-containing diet. The slides were stained with hematoxylin and eosin and graded according to the original modified Marsh classification [6]. The number of intraepithelial lymphocytes (IELs) per 100 villous epithelial cells was assessed by immunohistochemical staining. The upper limit of the reference interval in our laboratory was 25 IEL/100 epithelial cells. The number of eosinophils (EOS) per high-power fields (HPFs, $40 \mathrm{X}$ ) was also assessed; the upper limit of the reference interval in our laboratory was $60 \mathrm{EOS} / 10 \mathrm{HPFs}$.

\section{Elimination diet and double-blind placebo-controlled challenge method}

On entering the study all patients commenced a standard elimination diet, with the exclusion of wheat, cow's milk, eggs, tomato and chocolate [4]. Patients self-reporting food hypersensitivity were also asked to avoid ingestion and/or contact with the food(s) causing symptoms. After four weeks on the elimination diet they underwent DBPC wheat challenges, as previously described [4] (see Additional file 1). During all phases of the study, including the challenge period, the severity of symptoms was recorded: the patients completed a $100 \mathrm{~mm}$ visual analog scale (VAS), with 0 representing no symptoms, which assessed overall symptoms and the specific symptoms they each reported. DBPC challenge for CMPs was performed using an identical method [4], at least four weeks before or after wheat challenge and when the VAS score was $<10$, on the elimination diet. The challenges were stopped when clinical reactions occurred (increase in VAS score $>30$ ) for at least two consecutive days (onset of abdominal discomfort or pain, associated with a change in stool frequency and/or appearance). The challenges were considered positive if the same symptoms which had been initially present reappeared after their disappearance on the elimination diet.

\section{Other laboratory examinations}

The nutritional status of each patient was defined at diagnosis by measuring the following laboratory indices: hemoglobin, serum albumin and triglycerides.

\section{Bone mineral density assessment}

BMD was assessed at baseline by Dual Energy X-Ray Absorptiometry (DXA), using a QDR Discovery Hologic DXA in the femoral neck and in the lumbar spine by total body DXA. For each scan, BMD (expressed as absolute values in $\mathrm{g} / \mathrm{cm}^{2}$ ) and T-scores were recorded (for 
details see Additional file 1). Repeated measurements of the lumbar spine of ten healthy volunteers were performed to evaluate the in vivo precision of the absorptiometer. The average coefficient of variation was $2.1 \%$.

\section{Physical examination and dietary assessment}

At the start of the study, the BMI of all participants was recorded. The subjects also filled in a health and lifestyle questionnaire considering menarche, medical history and lifestyle habits (including physical activity, smoking and elimination diet, that is, wheat and other foods). The subjects also received a previously validated dietary form containing a printed list of the most common foods [7] (for details see Additional file 1). Final forms were analyzed using a computerized database and calcium intake was calculated using a nutrient composition database [8].

\section{Statistical analysis}

Data were expressed as mean \pm standard deviation (SD) when the distribution was Gaussian and differences were calculated using Student's $t$-test. Otherwise, data were expressed as median and range, and analyzed with the Kruskall Wallis test and with the Mann-Whitney $U$ test.
Fisher's exact and Mantel-Haenszel tests, Pearson's correlation and Spearman's rank correlation were used where appropriate. An analysis of variance (ANOVA) test was performed to evaluate the BMD differences between the three study groups; post hoc analysis and comparison between two groups was performed by means of the Bonferroni test. Multiple regression analysis was performed to evaluate the association between the presence of osteopenia and osteoporosis with the other clinical variables evaluated. $P<0.05$ was considered significant. All analyses were performed using the SPSS software package (version 16.0; Chicago, IL, USA).

The study was approved by the local Ethics Committee of the University Hospital of Palermo, and informed consent was obtained from all subjects.

\section{Results}

During the study period, 80 patients fulfilled the inclusion criteria, but 5 of them refused to undergo DXA and were excluded (see Additional file 2). The 75 patients included showed IBS-like symptoms on entering the study, which disappeared completely on the elimination diet and reappeared on DBPC wheat-challenge (VAS score increase $>30$ ) (see Additional file 3). Table 1 provides the

Table 1 Clinical characteristics of the NCWS patient group, compared to the control group composed of patients with IBS unrelated to NCWS and of CD patients

\begin{tabular}{|c|c|c|c|c|}
\hline Variables & $\begin{array}{l}\text { NCWS Patients } \\
\text { (Number }=75 \text { ) }\end{array}$ & $\begin{array}{l}\text { IBS Patients } \\
\text { (Number }=65 \text { ) }\end{array}$ & $\begin{array}{l}\text { CD Patients } \\
\text { (Number }=50 \text { ) }\end{array}$ & $P$ value \\
\hline Sex & $12 \mathrm{M}, 63 \mathrm{~F}$ & $10 \mathrm{M}, 55 \mathrm{~F}$ & $8 \mathrm{M}, 42 \mathrm{~F}$ & n.s. \\
\hline Age range (median) & 20 to 49 years ( 36 years) & 19 to 50 years ( 35 years) & 20 to 50 years ( 35 years) & n.s. \\
\hline \multicolumn{5}{|l|}{ IBS type } \\
\hline Diarrhea & $45(60 \%)$ & $36(55 \%)$ & 20 'typical' & \\
\hline Constipation & $7(9 \%)$ & $7(11 \%)$ & 30 'atypical' & n.s. \\
\hline Alternate bowel movements & $23(31 \%)$ & $22(34 \%)$ & & \\
\hline Symptom duration median (range) & 5 years (1 to 30 years) & 4 years ( 0.5 to 20 years) & 4 years ( 0.5 to 10 years) & n.s. \\
\hline \multirow[t]{3}{*}{ Weight loss } & $24 / 75(29 \%)$ & $3 / 65(4 \%)$ & $15 / 50(30 \%)$ & NCGS vs. IBS 0.0003 \\
\hline & & & & CD vs. IBS 0.0001 \\
\hline & & & & CD vs. NCGS n.s. \\
\hline \multirow[t]{3}{*}{ Anemia ( $\mathrm{Hb}<12 \mathrm{~g} / \mathrm{dl})$} & $12 / 75(16 \%)$ & $4 / 65(6 \%)$ & $25 / 50(50 \%)$ & NCGS vs. IBS 0.02 \\
\hline & & & & CD vs. IBS 0.0001 \\
\hline & & & & CD vs. NCGS 0.01 \\
\hline \multirow[t]{3}{*}{ Family history of CD } & $18 / 75(24 \%)$ & $0 / 65(0 \%)$ & $10 / 50(20 \%)$ & NCGS vs. IBS 0.0002 \\
\hline & & & & CD vs. IBS 0.001 \\
\hline & & & & CD vs. NCGS n.s. \\
\hline \multirow[t]{3}{*}{ Coexistent atopy } & 23/75 (30\%) & $6 / 65(9.5 \%)$ & $10 / 50(20 \%)$ & NCGS vs. IBS 0.01 \\
\hline & & & & CD vs. IBS n.s. \\
\hline & & & & CD vs. NCGS n.s. \\
\hline
\end{tabular}

Weight loss was considered when the patients reported a loss $>10 \%$ of body weight during the previous year. Family history of $C D$ indicates a CD diagnosis in a first-degree relative. Atopic diseases were: rhinitis, conjunctivitis, bronchial asthma, atopic dermatitis. CD, celiac disease; F, female; Hb, hemoglobin; IBS, irritable bowel syndrome; M, male; NCGS, non-celiac gluten sensitivity; NCWS, non-celiac wheat sensitivity; n.s., not significant; vs., versus. 
clinical characteristics of the NCWS patient group, compared to the control groups. NCWS patients had higher frequencies of weight loss, anemia (90\% had iron deficiency anemia), coexistent atopic diseases and family history of CD than IBS controls.

According to the results of the DBPC challenge with CMP, 30 of the 75 NCWS patients were found to have multiple food sensitivity (NCWS plus CMP sensitivity). These 30 patients were asymptomatic on the elimination diet and experienced IBS symptoms again on CMP challenge. Moreover, eight of these thirty patients experienced IBS-like symptoms after open challenges with egg (four cases), tomato (three cases) or chocolate (two cases).

HLA DQ2 or DQ8 haplotypes were present in 31 of 75 (41\%) NCWS patients. The duodenal histology showed normal villi but a high number of IEL in the mucosa (Marsh 1) in 39 NCWS patients (52\%), whereas the remaining $36 \mathrm{NCWS}$ patients had completely normal mucosa (Marsh 0); an eosinophil infiltrate was observed in 24 of the 75 (32\%) NCWS patients.

Figure 1 shows the individual values of BMD in the three study groups. NCWS patients had a significantly lower BMD than IBS controls both at the lumbar spine and at the femoral neck $(P<0.0001)$. The patients suffering from CD showed a significantly lower BMD than NCWS patients and IBS controls both at the lumbar spine (ANOVA: $\mathrm{F}=8.7 ; P<0.0001$; Bonferroni $P<0.0001$ ) and at the femoral neck (ANOVA: $\mathrm{F}=6.7 ; P<0.002$; Bonferroni: $P<0.0001$ ).

Figure 2 shows the percentage of patients of the three groups who had normal BMD, osteopenia or osteoporosis, evaluated both at the femoral neck and at the lumbar spine. The frequency of osteopenia and osteoporosis increased from IBS to NCWS, and to CD patients $(P<0.0001$, Spearman's rank correlation). Osteopenia or osteoporosis was found in $28 \%$ of the NCWS patients at the femoral neck and in $34 \%$ at the lumbar spine, whereas only $6 \%$ (lumbar spine) to $8 \%$ (femoral neck) of IBS patients had osteopenia and none of them had osteoporosis (for femoral neck $P<0.02$, chi-square 7.8; for lumbar spine $P<0.001$, chi-square 13). In total, 35 NCWS patients (46.6\%) had osteopenia or osteoporosis at one of the two sites (femoral neck or lumbar spine). CD patients had a higher frequency of osteopenia (50\%) and osteoporosis (14\%) than both NCWS and IBS patients.

Table 2 shows the frequency of some clinical, histological and genetic findings in the NCWS patients with and without reduced BMD. In patients with NCWS and osteopenia or osteoporosis, in comparison to the patients with normal BMD, there was a significantly higher frequency of subjects with BMI values $<20$ (Mantel-Haenszel $=5.7, P<0.02$ ), and with hemoglobin values $<12 \mathrm{gr} / \mathrm{dl}$ (Mantel-Haenszel $=10.1, P<0.001$ ), and of subjects with multiple food sensitivity (Mantel-

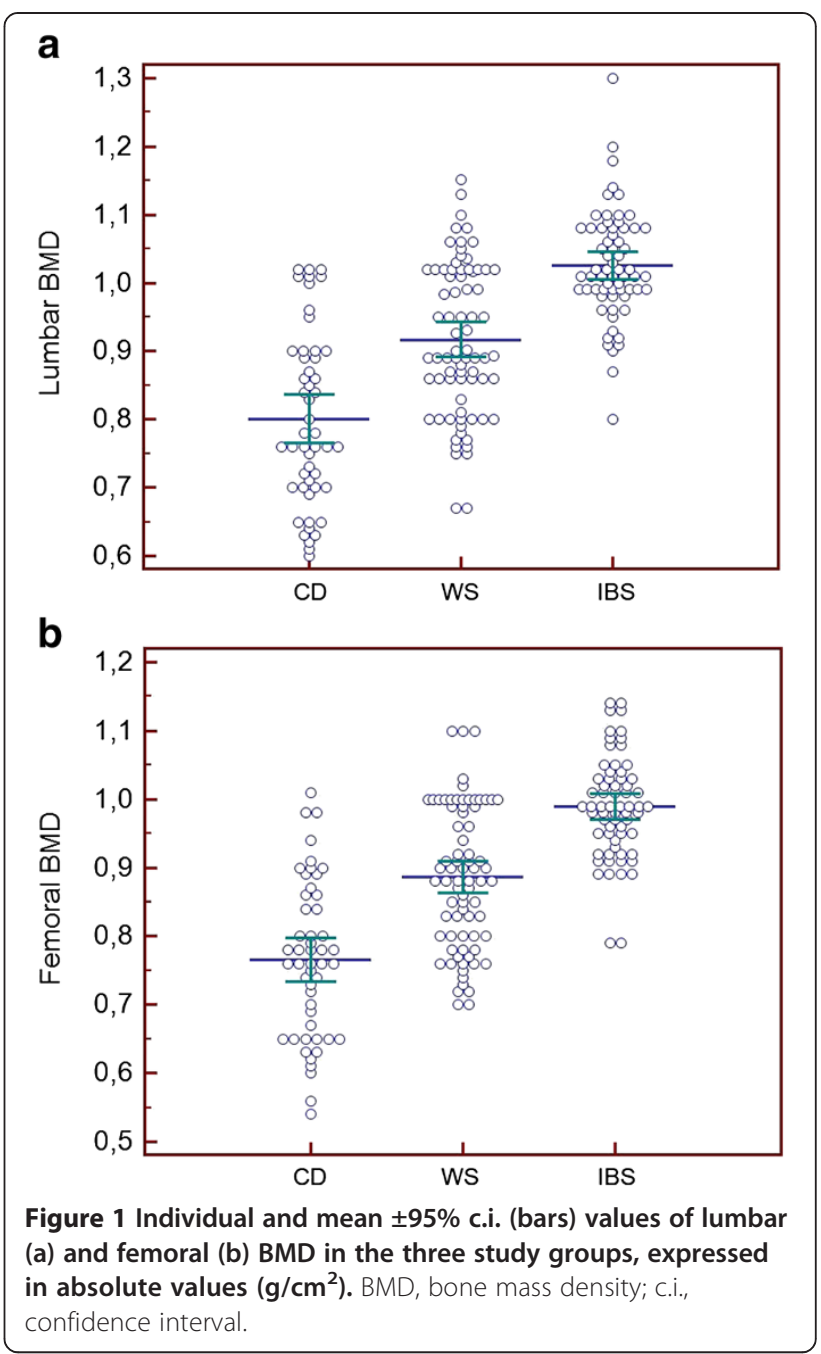

Haenszel $=7.8, P<0.005)$. The presence of osteopenia and osteoporosis did not correlate with the duodenal histology findings (Marsh 0 or Marsh 1 and presence/ absence of eosinophil infiltration), nor with the presence of the DQ2 or DQ8 alleles, or with the serum level of albumin and triglycerides (data not shown).

Furthermore, in NCWS patients, there was a significant direct correlation between the individual values of $\mathrm{BMD}(\mathrm{g} / \mathrm{cm} 2)$ and the BMI values $(P<0.0001)$ (Figure 3$)$. A similar, although less significant, correlation between BMD and BMI values was also found in IBS patients $(\mathrm{r}=0.30, P<0.04)$ and in $\mathrm{CD}$ controls $(\mathrm{r}=0.36, P<0.01)$.

Multiple regression analysis showed that the presence of osteopenia or osteoporosis was related exclusively to the BMI $(P<0.002)$ and to multiple food sensitivity $(P<0.03)$.

Mean values \pm SD of daily dietary calcium intake in NCWS patients (considered as a group) were $615 \pm$ $274 \mathrm{mg}$, significantly lower than in IBS controls $(766 \pm$ $284 \mathrm{mg} ; P<0.001)$ and in CD controls $(821 \pm 312 \mathrm{mg}$; 

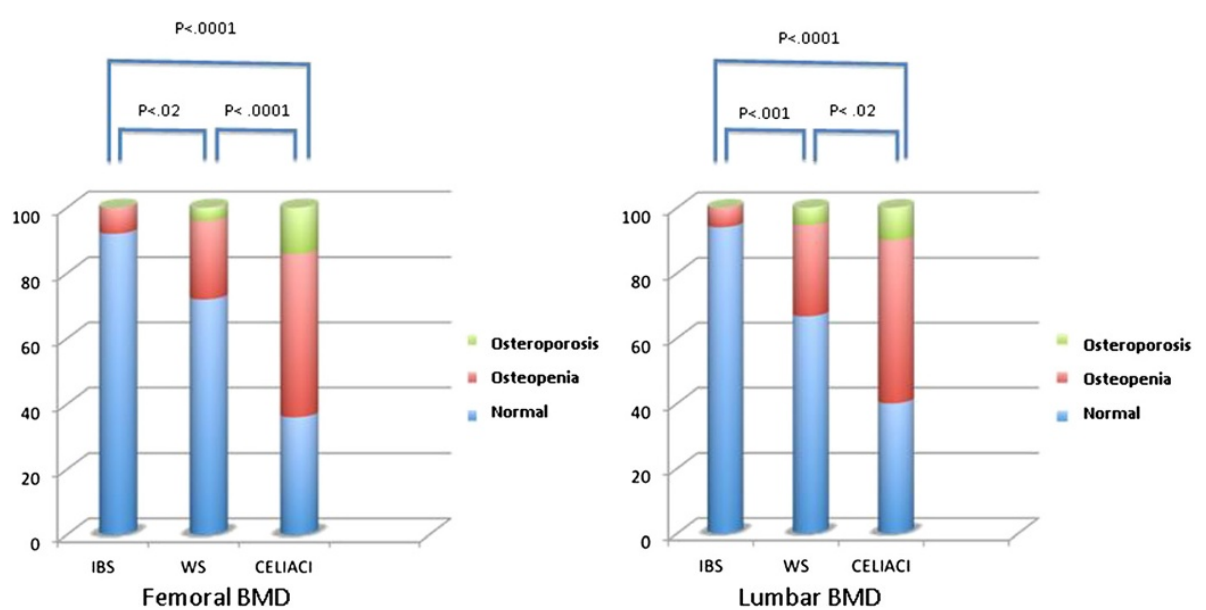

Figure 2 Percentage of the patients of the three groups who had normal BMD, osteopenia or osteoporosis, evaluated both at the lumbar spine and femoral neck. The frequency of osteopenia and osteoporosis was significantly different in the three groups, increasing from IBS to NCWS, and to CD patients ( $P<0.0001$, Spearman's rank correlation). BMD, bone mass density; $C D$, celiac disease; IBS, irritable bowel syndrome; NCWS, non-celiac wheat sensitivity.

$P<0.001)$. There was a significant difference between patients with NCWS alone (calcium intake: median $770 \mathrm{mg} /$ daily, range 300 to $1100 \mathrm{mg}$ ) and those with multiple food sensitivity (median $330 \mathrm{mg} /$ daily, range 30 to $900 \mathrm{mg}$ ) ( $\mathrm{z}=28.8, P<0.0001$, Mann-Whitney test).

Wheat exclusion from the diet before entering the study was self-reported in 21 of the 35 (60\%) NCWS patients with osteopenia/osteoporosis and in 16 of the 40 (40\%) patients without it $(P<0.08$, chi-square test). There was no difference in smoking habits or the levels of physical activity between the NCWS and IBS groups or within the NCWS group in the subjects with bone mass loss and those with normal bone mass.

\section{Discussion}

An increasing number of papers have recently been published by many groups which seemed to confirm that 'gluten-sensitivity' should definitely be included in the spectrum of gluten-related disorders [2,3]. However, some doubts persist about the pathogenic mechanisms [9] and it has been suggested that wheat could cause gastrointestinal symptoms independently of its gluten- content [10-13]. For these reasons we suggested the term 'non-celiac wheat-sensitivity' [14].

A recent UK study showed that the self-reported prevalence of wheat-sensitivity was 13\% [15]. The prevalence of strict adherence to a gluten-free diet in the general population ranged between 0.55 and $3.7 \%[15,16]$.

However, nothing is known about the osteoporotic risk in this condition, whereas it is known that more than $75 \%$ of untreated celiac patients suffer from a loss of bone mass [1].

We showed that the NCWS patients had a significantly lower bone mineral content than IBS controls both at the lumbar spine and at the femoral neck. Furthermore, 35 of the 75 NCWS patients $(46.6 \%)$ had osteopenia or osteoporosis at one of the two sites. This high frequency of low bone mass in NCWS patients is much more relevant if it is considered that our study population included young subjects, mostly premenopausal women, with median age 36 years. These women will reach the age of menopause - the most critical period for bone loss risk - with bone mass already reduced.

Table 2 Distribution of some variables in NCWS patients with normal bone density, with osteopenia and with osteoporosis

\begin{tabular}{lllllll}
\hline & BMI $<\mathbf{2 0}$ & Hb $<\mathbf{1 2}$ & DQ2/DQ8 & Marsh 1 & Duodenal eosinophils & MFS \\
\hline Normal $\mathrm{n}=40$ & $19(47 \%)$ & $2(5 \%)$ & $22(55 \%)$ & $24(60 \%)$ & $15(37 \%)$ & $10(25 \%)$ \\
Osteopenia $\mathrm{n}=29$ & $17(59 \%)$ & $7(24 \%)$ & $4(14 \%)$ & $12(41 \%)$ & $9(31 \%)$ & $16(55 \%)$ \\
Osteoporosis $\mathrm{n}=6$ & $6(100 \%)$ & $3(50 \%)$ & $5(83 \%)$ & $3(50 \%)$ & $0(0 \%)$ & $4(67 \%)$
\end{tabular}

MI: $\times 2 \mathrm{t}=5.7, P<0.02 ; \mathrm{Hb}: \mathrm{X} 2 \mathrm{t}=10.1, P<0.001 ;$ multiple food sensitivity: $\mathrm{X} 2 \mathrm{t}=7.8, P<0.005 ; \mathrm{HLA}$ DQ2/DQ8: $\mathrm{X} 2 \mathrm{t}=1.2, P=\mathrm{ns} ;$ Marsh 1: $\times 2 \mathrm{t}=1.4, P=\mathrm{ns} ;$ Duodenal eosinophils: $\mathrm{X} 2 \mathrm{t}=2.5, P=\mathrm{ns}$. Duodenal lymphocytosis (Marsh 1) was defined as a number of CD3+ $>25 \mathrm{IEL} / 100$ epithelial cells. Duodenal eosinophils infiltration was defined as a number of eosinophils $>60$ per 10 high-power fields. BMI, body mass index; Hb, hemoglobin; IEL, intraepithelial lymphocytes; NCWS, non-celiac wheat sensitivity, MFS, multiple food sensitivity. 

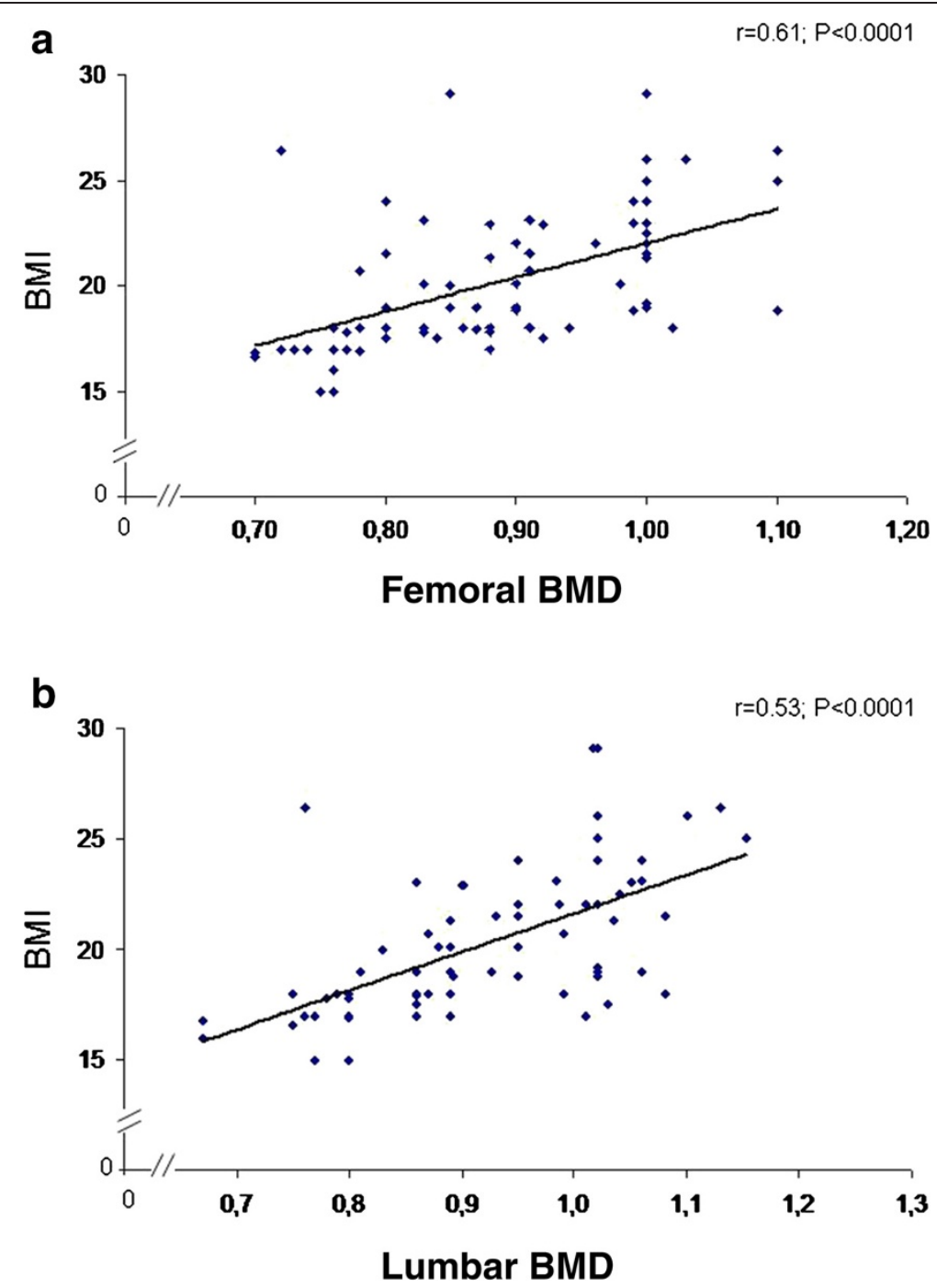

Figure 3 Individual values of BMD, expressed as absolute values $\left(\mathrm{g} / \mathrm{cm}^{2}\right)$, in relation to the $\mathrm{BMI}$, both at the femoral neck (Panel a) and at the lumbar spine (Panel b). A significant direct correlation between the individual values of BMD $(\mathrm{g} / \mathrm{cm} 2)$ and the BMI values $(P<0.0001)$ has been proved. BMD, bone mass density; BMI, body mass index.

Concerning the possible causes of osteopenia/osteoporosis in NCWS, we did not find any correlation with the degree of inflammation of the duodenal mucosa: there was no association with the Marsh 1 lesion (increased number of IELs) nor with the mucosal eosinophil infiltrate. The presence of the DQ2 or DQ8 HLA haplotypes was not associated with the presence of bone mass loss. On the whole these data seem to indicate that the mechanisms which cause osteoporosis in CD patients (malabsorption, inflammation, and so on) would not be pivotal in NCWS patients.

On the contrary, we found a direct correlation of bone mass loss with the BMI and hemoglobin values and these data could support the hypothesis of a role for malnutrition. In particular, it must be emphasized that about one third of our patient population reported weight loss of $>10 \%$ of body weight during the last year, and that $42 \mathrm{NCWS}$ patients (56\%) had a BMI less than 20. Thus, the role of malnutrition seems very important in our study group.

We found that the dietary calcium intake of the NCWS patients (mean $615 \mathrm{mg} /$ day) was significantly lower than that of the IBS controls, and much lower than the $1,000 \mathrm{mg} /$ day commonly recommended. The selective malnutrition could be caused by self-restriction of the diet: 37 of the 75 NCWS patients reported selfexclusion of wheat before entering the study, and dietary calcium intake was significantly lower in patients with multiple food sensitivity than in patients with NCWS alone, probably because the latter patients excluded a higher number of foods from their diet. Bucci et al. reported 70\% of NCWS patients excluded milk and dairy products from their diets to avoid symptoms [17]; 
Tavakkoli et al. reported that the people self-diagnosed as 'wheat-sensitives' had additional food avoidances including dairy $(59 \%)$ [18]. A lower percentage (35\%) of coexistent food intolerance has been recently reported in an Italian multicenter study [19]. This suggests that NCWS patients need dietary support to avoid mineral or other nutrient deficiencies.

However, the limits of our study must be highlighted. We did not suggest a low fermentable oligo-di-mono-saccharides and polyols (FODMAPs) diet to our patients, and it has been demonstrated that there was no evidence of effect of gluten in patients with gluten-sensitivity placed on diets low in FODMAP [13]. However, our patients became asymptomatic on the elimination diet and clearly reacted on DBPC wheat challenge, performed with a relatively small challenge dose of wheat. It is unlikely that our challenge method permitted us to identify many patients who are intolerant of the carbohydrate content of wheat, specifically fructans. Consequently, it is probable that we have studied a selected population which was different from that reported by Biesiekierski et al. [13]. Thus, our finding of the high prevalence of reduced bone mass and low BMI in NCGS patients must not be generalized.

We found a very high percentage of malnourished patients in our study group, but eating disorders were not regularly excluded by means of psychometric methods. In any case, the high percentage of NCWS patients with low BMI in our series could have influenced the high frequency of low BMD we found.

Furthermore, we cannot exclude that a percentage of our patients were in a 'pre-celiac condition', as could be suggested by the presence of DQ2/DQ8 haplotypes (41\%), CD family history (24\%) and duodenal lymphocytosis $(52 \%)$. However, these are common findings in the NCWS population [2].

We were unable to provide data about Vitamin D levels, parathyroid hormone and markers of both bone synthesis and re-absorption; future studies need to clarify the possible mechanisms of osteoporosis in NCWS.

\section{Conclusions}

We found an elevated frequency of bone mass loss in NCWS patients (46.6\%). This was related to low BMI and was more frequent in patients with NCWS associated with other food sensitivity than in patients with NCWS alone. Dietary support should be strongly recommended at the time of NCWS diagnosis, whatever is its pathogenesis.

\section{Additional files}

Additional file 1: Methods and diagnostic criteria in NCWS diagnosis. Additional file 2: Number of patients included/excluded during the different phases of the study.
Additional file 3: VAS score comparison in patients suffering from wheat sensitivity alone (GROUP 1) and patients suffering from multiple food hypersensitivity (GROUP 2), under challenge with wheat, cow milk and placebo.

\section{Abbreviations}

AGA: anti-gliadin; anti-tTG: anti-transglutaminase; BMD: bone mass density; BMI: body mass index; CD: celiac disease; CMP: cow's milk proteins;

DBPC: double-blind placebo-controlled; DXA: dual energy $x$-ray absorptiometry; EmA: anti-endomysium; EOS: eosinophils; HPFs: high-power fields; IBS: irritable bowel syndrome; IELs: intraepithelial lymphocytes; MFS: multiple food sensitivity; NCGS: non-celiac gluten sensitivity; NCWS: non-celiac wheat sensitivity; VAS: visual analog scale.

\section{Competing interests}

The authors declare that they have no competing interests.

\section{Authors' contributions}

AC: designed the research study and wrote the paper. AD'A: collected the data and revised the manuscript. MS: performed the statistical analysis and revised the manuscript. CS: performed the endoscopy study. Gl: collected the data and revised the manuscript. GG: performed the endoscopy study. IB: performed the immunologic assays. AS: collected the data. FA: collected the data. MC: collected the data. PM: collected and analyzed the data; revised the manuscript. All authors read and approved the final manuscript.

Authorship statement

Guarantor of the article: Professor Antonio Carroccio.

\section{Acknowledgements}

Thanks to native English speaker Frank Adamo for revising the text.

\section{Financial support}

This study was in part financed by the Italian Foundation for Celiac Disease Grant for Project 0132014 and in part by the University of Palermo FFR 2012 project "Gluten sensitivity and Irritable Bowel Syndrome".

\section{Author details}

'Department of Internal Medicine, University Hospital of Palermo, Via del Vespro 141, Palermo, Italy. ${ }^{2}$ Department of Internal Medicine, Giovanni Paolo II Hospital, Via Pompei, Sciacca, Italy. ${ }^{3}$ Department of Surgery, Oncology, and Odontology, University of Palermo, Via L. Giuffrè 5, Palermo, Italy. ${ }^{4}$ Pediatric Gastroenterology, Di Cristina Hospital Palermo, Piazza P. Montalto 2, Palermo, Italy. ${ }^{5}$ Department of Pathology, Buccheri - La Ferla Hospital Palermo, Via Messina Marine 197, Palermo, Italy.

\section{Received: 22 August 2014 Accepted: 7 November 2014}

\section{References}

1. Corazza GR, Di Sario A, Cecchetti L, Tarozzi C, Corrao G, Bernardi M, Gasbarrini G: Bone mass and metabolism in patients with coeliac disease. Gastroenterology 1995, 109:122-128.

2. Sapone A, Bai JC, Ciacci C, Dolinsek J, Green PH, Hadjivassiliou M, Kaukinen K, Rostami K, Sanders DS, Schumann M, Ullrich R, Villalta D, Volta U, Catassi C, Fasano A: Spectrum of gluten-related disorders: consensus on new nomenclature and classification. BMC Med 2012, 10:13.

3. Catassi C, Bai JC, Bonaz B, Bouma G, Calabrò A, Carroccio A, Castillejo G, Ciacci C, Cristofori F, Dolinsek J, Francavilla R, Elli L, Green P, Holtmeier W, Koehler P, Koletzko S, Meinhold C, Sanders D, Schumann M, Schuppan D, Ullrich R, Vécsei A, Volta U, Zevallos V, Sapone A: Fasano: non-celiac gluten sensitivity: the new frontier of gluten related disorders. Nutrients 2013, 5:3839-3853.

4. Carroccio A, Mansueto P, lacono G, Soresi M, D'Alcamo A, Cavataio F, Brusca I, Florena AM, Ambrosiano G, Seidita A, Pirrone G, Rini GB: Non-celiac wheat sensitivity diagnosed by double-blind placebo controlled challenge: exploring a new clinical entity. Am J Gastroenterol 2012, 107:1898-1906. 
5. Thompson WG, Longstreth GF, Drossman DA, Heaton KW, Irvine EJ, Müller-Lissner SA: Functional bowel disorders and functional abdominal pain. Gut 1999, 45:43-47.

6. Rostami K, Kerckhaert J, Tiemessen R, von Blomberg BM, Meijer JW, Mulder CJ: Sensitivity of antiendomysium and antigliadin antibodies in untreated celiac disease: disappointing in clinical practice. Am J Gastroenterol 1999, 94:888-894.

7. Carroccio A, Montalto G, Cavera G, Notarbatolo A: Lactose intolerance and self-reported milk intolerance: relationship with lactose maldigestion and nutrient intake. J Am Coll Nutr 1998, 17:631-636.

8. Istituto Nazionale della Nutrizione (Italian Institute for Nutrition): Food Composition Tables. Rome; 1986.

9. Mooney PD, Aziz I, Sanders DS: Non-celiac gluten sensitivity: clinical relevance and recommendations for future research. Neurogastroenterol Motil 2013, 25:864-871

10. Biesiekierski JR, Peters SL, Newnham ED, Rosella O, Muir JG, Gibson PR: No effects of gluten in patients with self-reported non-celiac gluten sensitivity after dietary reduction of fermentable, poorly absorbed, short-chain carbohydrates. Gastroenterology 2013, 145:320-328.

11. Halmos EP, Power VA, Shepherd SJ, Gibson PR, Muir JG: A diet low in FODMAPs reduces symptoms of irritable bowel syndrome. Gastroenterology 2014, 146:67-75.

12. Junker Y, Zeissig S, Kim SJ, Barisani D, Wieser H, Leffler DA, Zevallos V, Libermann TA, Dillon S, Freitag TL, Kelly CP, Schuppan D: Wheat amylase trypsin inhibitors drive intestinal inflammation via activation of toll-like receptor 4. J Exp Med 2012, 209:2395-2408.

13. Carroccio A, Mansueto P, D'Alcamo A, lacono G: Non-celiac wheat sensitivity as an allergic condition: personal experience and narrative review. Am J Gastroenterol 2013, 108:1845-1852.

14. Carroccio A, Rini G, Mansueto P: Non-celiac wheat sensitivity is a more appropriate label than non-celiac gluten sensitivity. Gastroenterology 2014, 146:320-321.

15. Aziz I, Lewis NR, Hadjivassiliou M, Winfield SN, Rugg N, Kelsall A, Newrick L, Sanders DS: A UK study assessing the population prevalence of selfreported gluten sensitivity and referral characteristics to secondary care. Eur I Gastroenterol Hepatol 2014, 26:33-39.

16. DiGiacomo DV, Tennyson CA, Green PH, Demmer RT: Prevalence of gluten-free diet adherence among individuals without celiac disease in the USA: results from the Continuous National Health and Nutrition Examination Survey 2009-2010. Scand J Gastroenterol 2013, 48:921-925.

17. Ciacci C, Bucci C, Zingone F, Fortunato A, Napoli M, Morra A, lovino P: What non celiac gluten sensitive patients do not eat (beside gluten). Chicago: 15th International Celiac Disease Symposium; 2013:61. Abs book C304.

18. Tavakkoli A, Lewis SK, Tennyson CA, Lebwohl B, Green PH: Characteristics of patients who avoid wheat and/or gluten in the absence of Celiac disease. Dig Dis Sci 2014, 59:1255-1261.

19. Volta U, Bardella MT, Calabrò A, Troncone R, Corazza GR: An Italian prospective multicenter survey on patients suspected of having non-celiac gluten sensitivity. BMC Med 2014, 12:85.

doi:10.1186/s12916-014-0230-2

Cite this article as: Carroccio et al:: Risk of low bone mineral density and low body mass index in patients with non-celiac wheat-sensitivity: a prospective observation study. BMC Medicine 2014 12:230.

\section{Submit your next manuscript to BioMed Central and take full advantage of:}

- Convenient online submission

- Thorough peer review

- No space constraints or color figure charges

- Immediate publication on acceptance

- Inclusion in PubMed, CAS, Scopus and Google Scholar

- Research which is freely available for redistribution

Submit your manuscript at www.biomedcentral.com/submit
() Biomed Central 